\title{
A atividade conceito do front end da inovação: uma busca sistemática da literatura
}

\section{The concept activity of the front end of innovation: a systematic search of literature}

\author{
André de Oliveira Leite Especialista Gestão da Informação. Universidade Federal de Santa Catarina (UFSC) - Brasil. \\ andreredes@gmail.com. \\ Roberto Fabiano Fernandes Doutor em Engenharia e Gestão do Conhecimento. Universidade Federal de Santa Catarina \\ (UFSC) - Brasil. roberto.fernandes@faculdadecesusc.edu.br. \\ João Artur de Souza Doutor em Engenharia de Produção, professor do Departamento de Engenharia do \\ Conhecimento Universidade Federal de Santa Catarina, (UFSC), Brasil - jartur@gmail.com \\ Gertrudes Aparecida Dandolini Doutora em Engenharia de Produção, professora na Universidade Federal de Santa Catarina \\ (UFSC) \\ Florianópolis, SC, Brasil.E-mail: gtude@egc.ufsc.br
}

\section{RESUMO}

A inovação está consolidada como fator-chave para empresas de ponta no desenvolvimento de novos produtos. Muitos aspectos estão relacionados ao termo inovação, um deles é o entendimento de como é o seu processo. Desta maneira, no processo de inovação diversos autores explicam que as fases iniciais, chamada de Front End da inovação, é decisiva para que novos produtos tenham aceitação no mercado. Para tanto, o Conceito, que é uma das atividades do Front End, deve ter especial atenção, uma vez que é a última etapa antes que novos produtos sejam desenvolvidos. $O$ objetivo deste trabalho é revisar a literatura por meio da busca sistemática, com auxílio de duas bases de dados, onde o foco está voltado para a atividade Conceito. Com isso, espera-se identificar oportunidades de pesquisas sobre o assunto. Os resultados mostram que os trabalhos publicados se concentram em poucos países e que as publicações tiveram seu pico no ano de 2016. Assim, espera-se que este trabalho auxilie na discussão sobre o tema Conceito, dada sua importância ao Front End da inovação.

Palavras-chave: Front End da Inovação. FEl. Atividade Conceito. Processo de Inovação. Desenvolvimento de novos produtos.

\section{ABSTRACT}

Innovation is consolidated as a key factor for leading companies in the development of new products. Many aspects are related to the term innovation, one of them is the understanding its process. In this way, in the innovation process, several authors explain that the initial phases, called Front End of innovation, are decisive for new products to be accepted in the market. To do so, the Concept, which is one of the activities of the Front End, should receive special attention, as it is the last phase before new products are developed. The objective of this work is to review the literature through a systematic search, with the help of two databases, where the focus is on Concept. It is expected that it will help to identify research opportunities on the subject. The results show that the published works are concentrated in a few countries and that the publications peaked in the year 2016. Thus, this research may come as an aid in the discussion on the theme Concept, given its importance to the Front End of innovation.

Keywords: Front End of Innovation. FEl. Activity Concept. Innovation Process. Development of new products. 


\section{INTRODUÇÃO}

Diante de um mercado competitivo como o atual, onde é necessário que as empresas estejam preparadas para novos desafios, o termo inovação está consolidado como premissa básica para sobrevivência. Como destaca Brem e Voigt (2007) nenhuma empresa que busca o sucesso pode se dar ao luxo de aparecer em público sem usar essa palavra em sua comunicação.

Muitos fatores podem influenciar a necessidade de inovar, desde clientes com novas necessidades, velocidade do processo tecnológico, até a competição em um mercado global (TIDD; BESSANT 2015). Estes autores ainda afirmam que gerenciar a inovação pode ser definida como um processo e como tal, deve ser analisado e medido.

Baregheh, Rowley e Sambrook (2009) corroboram com a definição de inovação vista como um processo e acrescentam que neste caso possui várias etapas, onde as organizações direcionam ideias, com objetivo de avançar, competir e estabelecer diferenças competitivas.

Partindo desse entendimento Koen et al. (2001) descrevem como três as principais fases do processo de inovação. A fase inicial pode ser chamada de Fuzzy Front End(FFE), a segunda New Product Developement (NDP) e fase final de comercialização ou implementação. Para esses autores, o termo FFEvem da complexidade de gerir o Front End, em que as atividades muitas vezes ainda são caóticas, imprevisíveis e não estruturadas.

Seguindo esse prisma, Kim e Wilemon (2002) explicam que a fase inicial FFE, que posteriormente fora denominada de Front End da Inovação (FEl), representa um dos maiores desafios para os gestores de inovação. Fatores influenciadores no FEl como imprecisão tecnológica, de mercado e de recursos, bem como a qualidade incerta das ideias podem ter impacto direto no próximo estágio do processo (desenvolvimento de produtos), visto que aumenta a chances de reduzir a eficiência e pode terminar como um produto não bem-sucedido no mercado (Kim; Wilemon, 2002).

Para Koen et al. (2001) a fase inicial do FEl pode ser detalhada com as seguintes atividades: identificação de oportunidades; análise de oportunidades; geração de ideias; seleção de ideias; e conceito.

Para Ayağ (2016) selecionar o melhor conceito entre um conjunto de alternativas disponíveis tem sido uma questão importante para as empresas. $O$ autor justifica sua análise, visto à medida que o desenvolvimento avança em um determinado conceito selecionado, torna-se mais difícil fazer alterações de design, pois interfere em termos de custo e cronograma.

Frishammar, Florén e Wincent (2011) apontam que muitas pesquisas mostraram a redução da incerteza como crítica no Front End da inovação, mas pouca atenção tem sido dada ao conceito igualmente importante para evitar falhas na etapa seguinte do processo de inovação.

Este artigo tem como objetivo revisar a literatura de forma sistemática, utilizando duas bases de dados, onde o foco está voltado para o FEl, mais precisamente na sua última atividade, o conceito. Também, com base na metodologia empregada, busca-se reunir materiais que tratam dessa etapa, a fim de saber o que está sendo pesquisado e obter uma definição mais precisa do termo conceito, uma vez que, segundo Koen et al. (2001) é a fronteira para o NDP.

A justificava desta pesquisa tem relevância pela importância do FEI nas fases seguintes do processo de inovação. Neste contexto, a atividade Definição do Conceito deve ser explorada e analisada com mais atenção, conforme explica Cao, Zhao e Nagahira (2011)

Para tanto, este material está organizado da seguinte maneira: além da presente seção intitulada como introdução, a segunda seção traz a revisão da literatura sobre o FEl. A terceira seção apresenta os procedimentos metodológicos que apoiaram esta pesquisa. A quarta seção aborda as discussões relacionadas aos diversos pontos de vistas dos autores pesquisados, e, por fim, a quinta seção apresenta as conclusões da pesquisa. 


\section{FRONT END DA INOVAÇÃO - FEI}

Koen et al. (2001) tratam o FEl como sendo início do processo de inovação, composto pelas atividades que vêm antes da parte formal e bem estruturada do desenvolvimento de novos produtos, onde a informação e o conhecimento são insumos importantes.

Os mesmos autores denominam as fases iniciais de Front Ende o detalha nas seguintes atividades: 1 ) Identificação de oportunidades; 2) Análise de oportunidades; 3) Geração de ideias; 4) Seleção de ideias; e 5) Conceito. A Figura 1, ilustra o processo de inovação, com destaque para a parte inicial do processo.

Figura 1 - Representação do Processo de Inovação

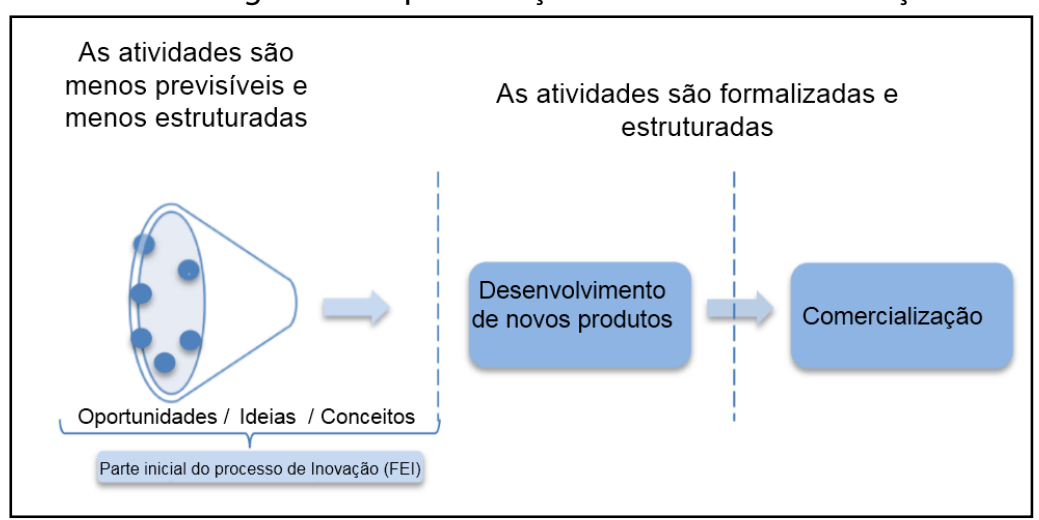

Fonte: adaptado de Koen et al. (2001).

A representação do processo de inovação descrito por Koen et al. (2001) é realizada de forma macro, ou seja, não há um detalhamento das informações e dos conhecimentos existentes no fluxo do processo, o que evidenciaria valor a todo processo.

Tidd e Bessand (2015) afirmam que se for comparado o Front End com a fase de desenvolvimento, ainda pouco se saberia sobre as atividades que constituem o mesmo. Isso se deve ao fato de o Front Endainda ser considerado o menos previsível e o menos estruturado, além do mais problemático no processo de inovação.

Essa afirmação, apesar de parecer negativa, tem o seu aspecto positivo, visto que, por ser considerado a fase mais crítica, representa também um potencial de ser explorado. e fornece maiores oportunidades para melhorar a capacidade global de inovação de uma empresa (ARTTO et al., 2011).

Essa afirmação já era corroborada por Takahashi e Takahashi (2007) que declararam que é nessa fase que ocorre o aumento substancial da probabilidade de se encontrar novos conceitos de novos produtos e negócios, no qual se formula o valor potencial para a inovação.

Elfvengren, Kortelainen e Tuominen (2009), Sandmeier et al. (2004) e Flint (2002) defendem a necessidade de estruturar e integrar as atividades do Front End para melhorar o desempenho da inovação e permitir o acesso às informações de várias fontes, resultando em formas mais viáveis de desenvolvimento de novos negócios.

Antonello, Romano e Martins (2015) afirmam que quando importantes decisões nas etapas iniciais do projeto são realizadas de forma não estruturada, a chance de decisões errôneas é maior, já que a etapa inicial do projeto tem a capacidade de influenciar a maioria da alocação de recursos e agregação de custos do produto.

Salmela, Santos e Happonen (2013), Gassmann e Schweitzer (2013) também defendem a necessidade de organizar a parte inicial do processo de inovação, e afirmam que, para isso, deve ser encontrado o justo equilíbrio entre o formalismo e a criatividade. Os autores salientam a importância de a criatividade ser guiada por meio de determinados processos formais, entretanto sem que a inibam.

Vários estudos empíricos têm demonstrado que a redução das incertezas técnicas e de mercado, bem como uma melhor planificação inicial do desenvolvimento de novo projeto tem um impacto positivo na 
inovação e aumenta a probabilidade de sucesso dos projetos (FRISHAMMAR et al., 2016). Essas incertezas são reduzidas em todas as dimensões quando informações suficientes são obtidas nas fases iniciais do Front End, para apoiar uma tomada de decisão.

Por essas razões é que as mudanças ocorridas na fase inicial, resultantes da mitigação das incertezas, impactam na redução de custos, muito mais que se fossem descobertas no final do projeto de inovação (STEVENS, 2014; THANASOPON; PAPADOPOULOS; VIDGEN, 2013; HERSTATT; VERWORN, 2001).

Os autores Zhao, Xiang e Yi (2017) citam que para diminuir a imprecisão atribuída a parte inicial do processo de inovação há necessidade de desenvolver e ampliar os relacionamentos com parceiros, apoiadores e alianças, que forneçam suporte organizacional para atividades do Front End.

\subsection{Atividades que compõem o Front End da Inovação}

As pesquisas com Front End começaram com grupo chamado PEN - Process Effectiveness Network do Instituto de Pesquisas Industriais (IRI) voltado para grandes empresas. Desta pesquisa resultou um novo framework para o Front End, chamado de New Concept Development Model - NCD model, que proveu uma linguagem comum e as práticas mais eficazes para otimizar o Front End da inovação.

Em 2002, outra pesquisa deu continuidade ao trabalho do grupo PEN. Para essa nova pesquisa, o NCD foi tomado como lente para identificar e analisar os atributos e atividades organizacionais que promovem o sucesso do Front End (KOEN; BERTELS; KLEINSCHMIDT, 2014). Para esta pesquisa foi utilizado um survey procurando estudar as atividades do Front End da Inovação nas empresas, bem como serviu de base para a coleta de dados realizada em uma entrevista denominada de "A Benchmarking Study of Front End of Innovation Activities and Performances"(SITE STEVENS INSTITUTE OF TECHNOLOGY, 2004).

Sobre o NCD, os autores desenvolveram uma construção teórica, a fim de proporcionar percepções e uma linguagem comum sobre as atividades iniciais do processo de desenvolvimento de produtos. Koen et al. (2001 e 2002), apresentam uma visão do Front End, por meio de um modelo composto de cinco elementos:

a) Identificação da oportunidade;

b) Análise da oportunidade;

c) Geração e aperfeiçoamento de ideias;

d) Seleção de ideias;

e) Desenvolvimento do conceito e da tecnologia.

Segundo os autores, a interação existente entre os cinco elementos é cíclica ou iterativa. A forma circular tem como intenção sugerir que as ideias devem fluir e iterar entre todos os cinco elementos.

Este modelo é o que trata com mais detalhes os elementos e atividades que fazem parte do Front End da Inovação. No entanto, os próprios autores sugerem que ele seja mais bem detalhado. Além da iteração, destaca-se a inter-relação entre as atividades do Front End. O modelo de Koen et al. (2001) tem seu foco no processo de inovação, com destaque a parte inicial do processo.

No que tange ao modelo NCD, os autores o formalizaram em três partes: motor, a roda e a borda. $\mathrm{O}$ motor (engine) é o elemento central que impulsiona os cinco elementos do Front End e é alimentado pela liderança e cultura da organização. A roda ( $w h e e$ ) é a parte interna do modelo, composta por cinco elementoschave que compreendem o Front End: 1) identificação de oportunidades, 2) análise de oportunidades, 3) a geração de ideias, 4) seleção de ideias, e 5) definição de conceito. O terceiro elemento do modelo NCD é a borda (border) e consiste nos fatores do ambiente externo que influenciam o motor e elementos do Front End. 
Figura 2 - Modelo NCD

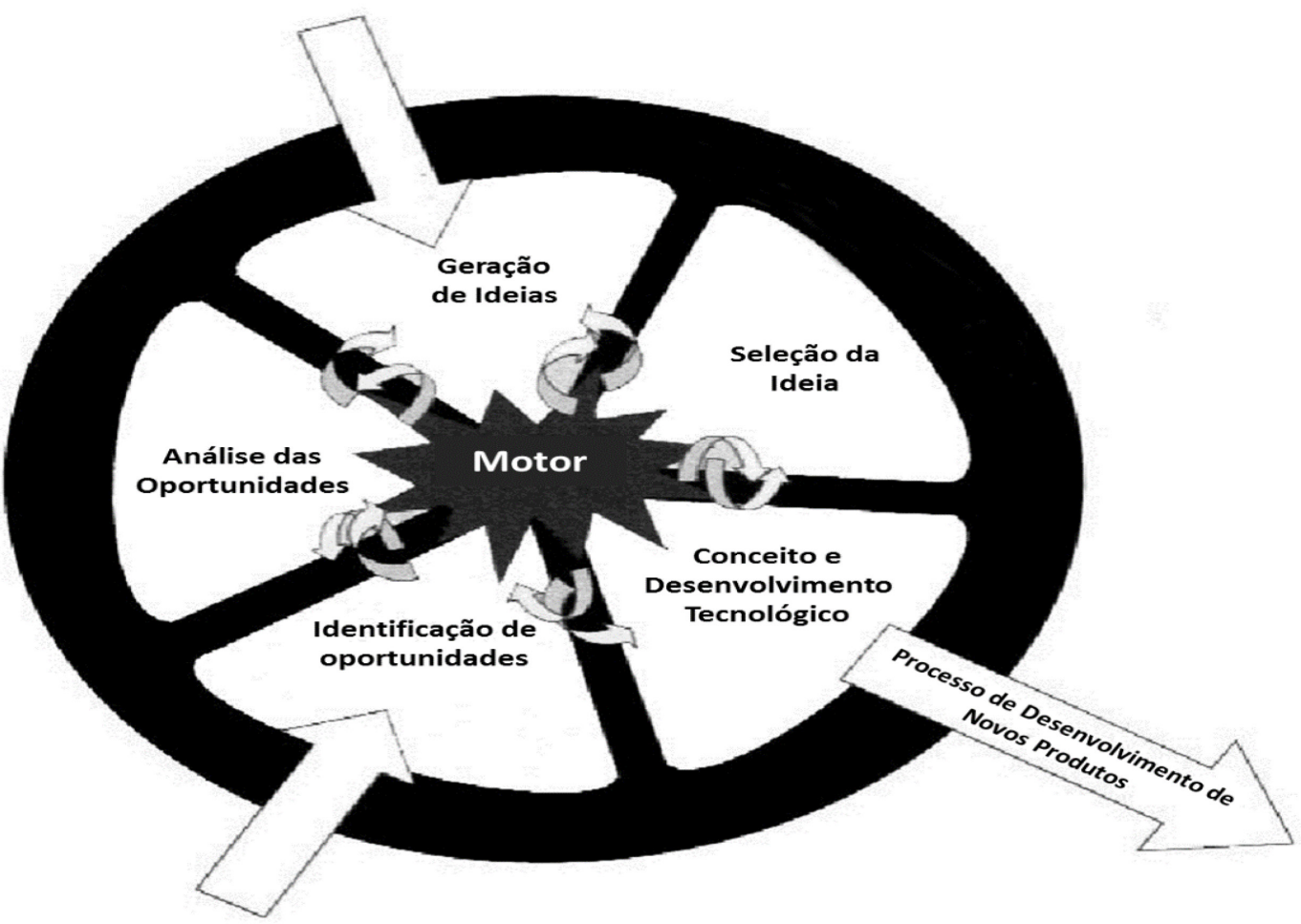

Fonte: Koen et al. (2001, p. 3, tradução nossa).

Sobre os cinco elementos-chave, Koen et al. (2001) os caracterizam da seguinte forma:

1) Identificação de Oportunidades: é o elemento que é acionado pelas metas de um negócio, projetos ou mesmo por padrão da empresa. É um processo formal e iterativo. Como exemplo, destaca-se que pode ser uma resposta em curto prazo a uma ameaça competitiva, uma forma para adquirir vantagem competitiva, ou um meio para simplificar, agilizar ou reduzir o custo das operações. A oportunidade também pode ser uma nova direção para o negócio ou uma pequena atualização para um produto existente.

2) A análise das oportunidades: se refere a como transformar as oportunidades identificadas em oportunidades de negócios. Também pode ser um processo formal e iterativo.

3) Geração de Ideias: refere-se ao nascimento, desenvolvimento e maturação da oportunidade para uma ideia concreta. Este representa um processo evolutivo em que as ideias são construídas, destruídas, combinadas, reformuladas, modificadas e atualizadas. Esse processo evolutivo acontece quando há o contato com clientes, na comunicação entre equipes multifuncionais, e na colaboração entre empresas, universidades e demais organizações. Destaca-se que a geração de ideias também pode alimentar o processo de identificação de oportunidades. Objetiva-se como saída uma descrição mais desenvolvida da ideia ou de um conceito de produto.

4) Seleção de Ideias: é a atividade crítica de escolha das ideias a fim de alcançar valor ao negócio. No entanto, a atividade crítica para a Seleção Ideias, assim como a Análise de Oportunidades, não deve impedir o crescimento e avanço devido à incerteza.

5) Definição de Conceito: é o elemento final e envolve o desenvolvimento de possíveis negócios com base em estimativas em potencial de mercado, necessidades do cliente, requisitos de investimento, avaliações de concorrentes e risco do projeto. Pode ser considerada a fase inicial do processo de desenvolvimento de produto.

Com elementos-chave definidos por Koen et al. (2001), na próxima seção os autores que compõe o portfólio bibliográfico adotado neste trabalho descrevem aspectos relacionados ao conteúdo sobre a atividade conceito no Front End da inovação. 


\subsection{Atividade Conceito}

Conforme descrito anteriormente, a fase de definição do conceito é a fronteira para o desenvolvimento de novos produtos, e sua importância fica clara quando Ayağ (2016) indica que à medida que o desenvolvimento posterior avança em um conceito selecionado, torna-se mais difícil fazer alterações de design em termos de custo e cronograma. Portanto, está fase tem fundamental importância no Front End.

Produtos inovadores devem ser gerenciados nesta fase com cautela, e como alertam Gassmann e Schweitzer (2013), existem diversos problemas que podem ser causados devido à má implementação do Front End no processo de inovação.

Backman, Börjesson e Setterberg (2007) lembram a impossibilidade de definir um processo padrão satisfatórios para todos os tipos de conceitos, principalmente porque o contexto em que conceito está inserido é fundamental para sua definição. Em seu trabalho, Frishammar, Florén e Wincent (2011) apontam que muitas pesquisas mostraram a redução da incerteza como crítica no Front End da inovação, mas pouca atenção tem sido dada ao conceito igualmente importante para evitar falhas na etapa seguinte do processo de inovação.

Achiche et al. (2013) explicam que as empresas tendem a iniciar os estágios do Front End sem uma definição clara ou análise do processo para ir da identificação da oportunidade à geração do conceito, este fato tem como consequência que o processo Front Endé frequentemente abortado ou forçado a ser reiniciado.

Neste contexto, a atividade Definição do Conceito deve ser explorada e analisada com mais atenção e conforme explica Cao, Zhao e Nagahira (2011) quanto mais intensamente novos projetos forem planejados antes do início do desenvolvimento, tanto mais incertezas de mercado e técnicas serão reduzidas. Para Elverum e Welo (2014) ter um conceito efetivo é sinônimo de desenvolvimento de vários protótipos. Fato reforçado por Backman, Börjesson e Setterberg (2007) quando explica que existe a necessidade de distinguir entre diferentes conceitos e explorar seus vários pré-requisitos para obter sucesso.

Por outro lado, para que a Front End forneça um conceito adequado aos objetivos do negócio é necessário analisar todas suas atividades, e conforme explicam Duin et al. (2009), a análise dos métodos de geração de ideias levou a um conceito de jogo que pode ser usado para estimular a criatividade dos participantes no processo de inovação. Ainda no prisma de desenvolver ferramentas para o Front End, Chew (2016) apresenta o método design integrado para oferecer, entre outros atributos, conceito de serviços. Para Jevnaker, Tellefsen e Lüders (2015) a experimentação com uma abordagem colaborativa e assistida por designer de criação de conceito pode fornecer novos insights sobre a campo emergente de inovações de serviço.

A decisão por investir ou não em determinada ideia para gerar um conceito não é uma tarefa trivial, como explica lluz e Shtub (2015), este fato segundo os autores, está relacionado à complexidade das fases iniciais do desenvolvimento de novos produtos.

Neste sentido, Fox (2012) em seu artigo propôs melhorias nos processos de triagem do NDP com análise de problemas de sustentabilidade menos previsíveis que podem surgir através de produtos manufaturados, objetivando facilitar a redução de riscos e suas consequências negativas.

Barros et al. (2015) utilizam a técnica Delphi como metodologia para identificar as variáveis e os principais fatores críticos que existem durante a fase de conceito do produto. Uma alternativa vem do trabalho de Rocha, Souza e Filho (2014) que comprovaram a eficiência da prática da empresa em desenvolver simultaneamente múltiplos conceitos para cada mecanismo ou detalhe do projeto.

Além da definição de múltiplos conceitos outro fator importante é descrito por Herstatt et al. (2006) que explica a necessidade da integração de clientes e usuários para desenvolver ideias e conceitos de produtos.

Neste item foram exibidos diversos autores definidos no portfólio bibliográfico que abordaram atividade conceito. Na próxima seção serão definidos os procedimentos metodológicos que serviram como base para esta pesquisa. 


\section{PROCEDIMENTOS METODOLÓGICOS}

Para a busca dos dados desta pesquisa foram selecionadas as bases $\operatorname{Scopus}^{\oplus}$ e Proquest ${ }^{\oplus}$. A respeito da escolha da base de dados $S$ copus $^{\circ}$ se justifica por ser a maior base de resumos e referências bibliográficas de literatura científica revisada por pares, segundo informações contidas no site da Elsevier, em janeiro de 2015. Também se pode afirmar que a Scopus ${ }^{\circledR}$ permite uma visão multidisciplinar da ciência e integra todas as fontes relevantes para a pesquisa básica, aplicada e de inovação tecnológica através de patentes, fontes da web, mas com conteúdo científico, periódicos de acesso aberto, memórias de congressos e conferências.

No que se refere a base de dados ProQuest ${ }^{\oplus}$, utilizou-se especificamente a base de teses. É uma base multidisciplinar, considerada a maior e mais relevante no acesso a teses e dissertações na íntegra (texto completo) do mundo. É também o depósito oficial de teses e dissertações nos Estados Unidos, e mantido pela ProQuest para a Library of Congress norte-americana. Além disso, a ProQuest ${ }^{\circledR}$ tem acesso a importantes bancos de dados comerciais, permitindo ao pesquisador um diferencial na busca de artigos.

Para organização das buscas utilizou-se a ferramenta Endnote ${ }^{\oplus}$, que é um software comercial que possibilita ao pesquisador organizar, armazenar e usar referências bibliográficas.

Ainda sobre as bases, justifica-se que além de realizar uma busca exploratória, foram criados alertas que vêm mantendo a atualização da pesquisa até o presente momento.

Tanto a busca, como os alertas foram criados em julho de 2018 e seguiram os critérios para seleção dos artigos descritos no quadro um:

Quadro 1 - Critérios para seleção dos artigos

\begin{tabular}{|c|c|}
\hline Descrição & Resultado \\
\hline $\begin{array}{l}\text { A busca principal e os alertas têm as } \\
\text { seguintes palavras-chave: }\end{array}$ & $\begin{array}{l}\text { "concept and front end innovation", com as seguintes consultas: } \\
\text { ("concept AND (process OR steps) AND "front end of innovation" } \\
\text { "), ("concept" AND "Front End innovation"), (fuzzy AND "front end } \\
\text { innovation"), (fuzzy AND "front end" AND concept AND } \\
\text { innovation), onde deixou-se livre a faixa temporal de busca por ano } \\
\text { de publicação, língua e tipo de documento resultante, quando } \\
\text { realizada na base Scopus. }\end{array}$ \\
\hline A seleção do artigo: & $\begin{array}{l}\text { Quando contenha ou no título, ou no resumo, ou nas palavras- } \\
\text { chave, a palavra-chave de busca. }\end{array}$ \\
\hline Tipo de documento: & Article e book chapter. \\
\hline Línguas: & Português e inglês. \\
\hline $\begin{array}{l}\text { Seleção de artigos disponíveis e que } \\
\text { contenham texto na íntegra: }\end{array}$ & $\begin{array}{l}\text { Na base Scopus foram encontrados } 169 \text { artigos. Os mesmos termos } \\
\text { foram utilizados na base Proquest, porém, como ela é uma base } \\
\text { onde há dissertações e teses com muitos materiais publicados } \\
\text { sobre o assunto, optou-se por buscar materiais que fossem } \\
\text { revisados por pares e que estivessem disponíveis na íntegra. Na } \\
\text { base Proquest encontrou-se } 394 \text { artigos, teses ou dissertações. } \\
\text { A busca foi realizada no dia } 02 / 07 / 2018, \text { por dois pesquisadores. } \\
\text { Ressalta-se que a busca vem sendo complementada por meio de } \\
\text { alertas, identificação de artigos clássicos sobre o tema e assuntos } \\
\text { correlatos envolvidos na pesquisa. } \\
\text { Sobre essa busca, obteve-se um total de } 563 \text { artigos pesquisados } \\
\text { nas bases de dados Scopus }{ }^{\oplus} \text {, } \text { ProQuest }{ }^{\oplus} \text {. }\end{array}$ \\
\hline $\begin{array}{l}\text { Para os } 563 \text { artigos, foram aplicados } \\
\text { filtros de leitura do título, palavras- } \\
\text { chave e resumo e verificando a } \\
\text { adequação do artigo ao tema de } \\
\text { pesquisa. Assim, chegou-se a um } \\
\text { portfólio de } 115 \text { artigos, para os } \\
\text { quais novamente aplicou-se filtros: }\end{array}$ & $\begin{array}{l}\text { 1) Considerar somente os artigos que estavam disponíveis para ser } \\
\text { baixados (realizar o download); } \\
\text { 2) Ler os artigos na íntegra. }\end{array}$ \\
\hline
\end{tabular}

Fonte: construído pelos autores (2018). 
O portfólio final, aderente ao tema concept and front end innovation ficou composto por 20 artigos, para os quais foi feita a análise crítica de cada artigo que será apresentada no tópico Discussão. A análise representa o conjunto restrito de publicações com reconhecimento e destaque científico, com título, resumo e conteúdo completo, alinhados com determinado tema segundo a percepção e delimitações de um pesquisador (TASCA, 2013).

\section{DISCUSSÃO}

Nesta seção serão verificados os resultados preliminares das buscas, o fator de impacto dos periódicos e as principais oportunidades de pesquisa identificadas nos artigos pesquisados.

\subsection{Resultados Preliminares das Buscas}

Conforme mencionado na seção três - Procedimentos metodológicos, procedeu-se a leitura e análise detalhada dos 20 artigos que compuseram o portfólio bibliográfico, pois foram considerados alinhados ao escopo do tema desta pesquisa, A Atividade Conceito no Front End da Inovação: uma Busca Sistemática da Literatura. Ainda, levantou-se a quantidade de publicações por ano, abordagens de pesquisa (qualitativa, quantitativa e mista), além das dos países onde foram realizadas as pesquisas.

Na Figura três, apresenta-se a quantidade de publicações por ano, bem como a evolução temporal das pesquisas sobre o tema desde 2001 (considerando as 20 publicações). Foi observado que $84 \%$ dos estudos são recentes, ou seja, foram realizados nos últimos 7 anos (2011 a 2017), com destaque para os anos de 2011, 2013, 2014, 2015 e 2016, somando 16 publicações (80\%).

Figura 3 - Quantidade de publicações por ano sobre o tema Conceito

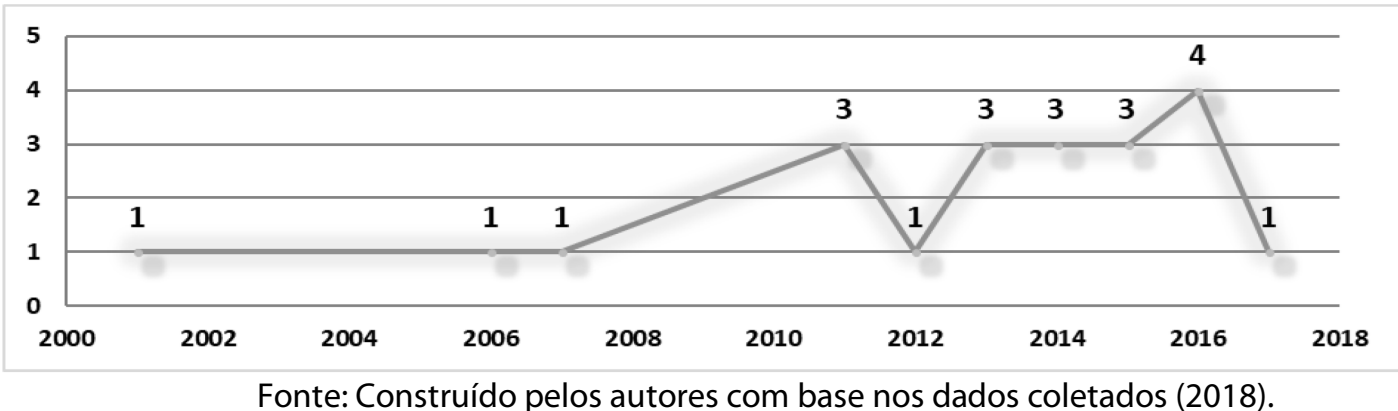

Outro aspecto analisado se refere a abordagem das publicações. Identificou-se que a maioria dos trabalhos são qualitativos. Para melhor apresentar os dados, a figura 4 ilustra a distribuição dos artigos conforme a abordagem.

Figura 4 - Classificação dos artigos por abordagem

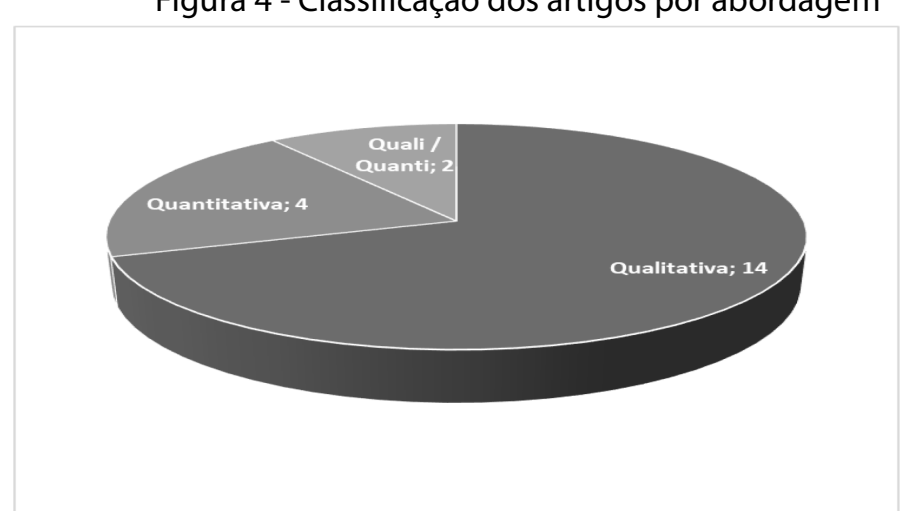

Fonte: Construído pelos autores com base nos dados coletados (2018). 
Verificou-se que trabalhos de natureza qualitativa têm sido predominantes, pois totalizou $70 \%$ dos casos classificados, ao passo que $20 \%$ são de natureza quantitativa e $10 \%$ utilizaram a abordagem mista.

Identificou-se os países onde as pesquisas foram realizadas e, como destaque, ressalta-se a participação do Brasil em duas publicações. Os Estados Unidos detêm a grande maioria das publicações de forma isolada, mas também há muitas publicações de países europeus (9), conforme pode-se observar na Figura 5.

Figura 5 - País origem da pesquisa

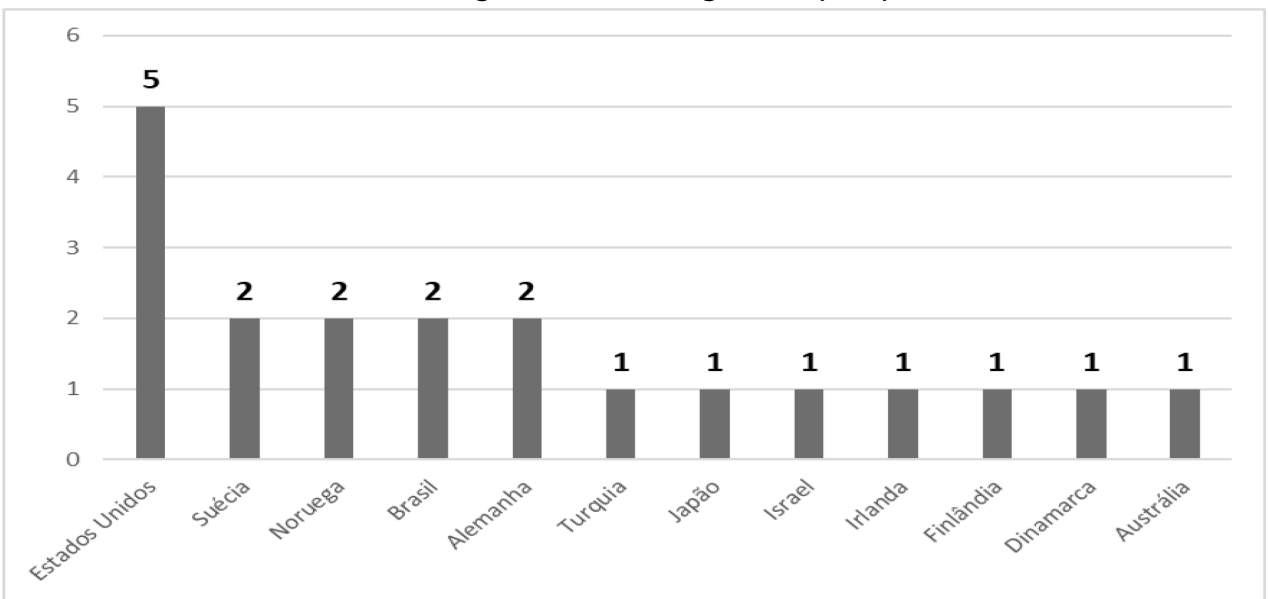

Fonte: Construído pelos autores com base nos dados coletados (2018).

\subsection{Fatores de impactos dos periódicos contidos no portfólio bibliográfico.}

Outra informação analisada, subsequentemente, foi o estrato do site ELSEVIER (2019). Para essa análise, cada um dos 20 periódicos, foram analisados considerando fatores de impacto e índice $\mathrm{H}$, visando analisar a qualificação das revistas.

Percebeu-se que metade das publicações (10) resultaram de envios a conferências, sendo assim não possuem qualificação pelo SJR.

Figura 6 - Qualificação do Journal

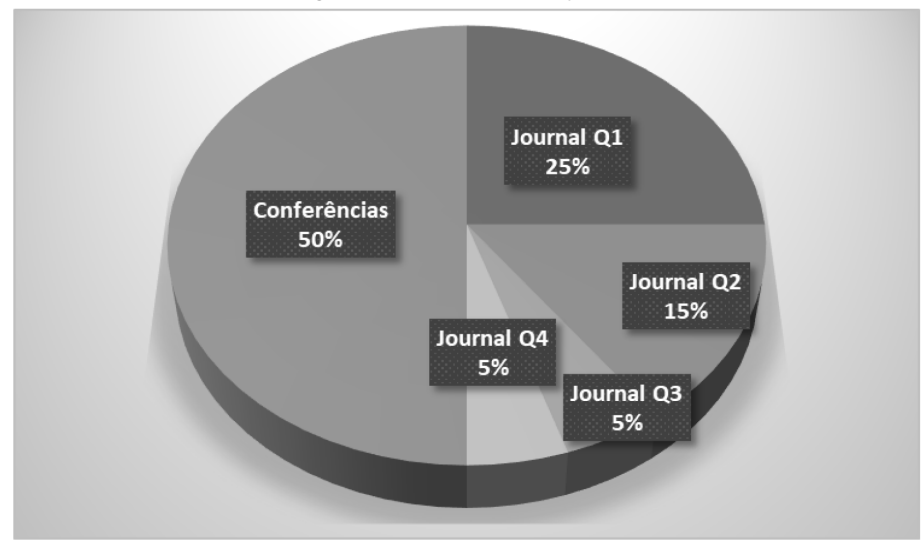

Fonte: Construído pelos autores com base nos dados coletados (2018)

Os Quartis Q1, Q2, Q3 e Q4 são usados para melhorar a comparabilidade entre publicações dentro de uma área e derivam da divisão por quatro da lista total de revistas indexadas para cada área.

Os artigos com qualificação Q1 e Q2 são os que publicaram em periódicos da área de Negócios e Gestão, Indústria e Engenharia de Manufatura, Estratégia e Gestão, Marketing e Sistemas de Informação, totalizando sete publicações. Duas publicações fazem parte de um livro específico sobre a estruturação do 
Front Ende dessas, apesar de não ter qualificação no SJR, obteve-se uma grande contribuição para atender os objetivos da pesquisa. Somente uma publicação teve qualificação Q4, mas isso se deve ao fato de ser um periódico novo.

Como análise final do portfólio bibliográfico foram levantadas as oportunidades para estudos futuros. Do portfólio bibliográfico, composto de 20 trabalhos, 14 artigos trazem, de forma explícita, recomendações de trabalhos futuros (Quadro 2). Isso vem a atender o objetivo principal da pesquisa em mostrar a situação atual da literatura e identificar oportunidades de pesquisas sobre o assunto.

\subsection{Principais oportunidades de pesquisa do Portfólio Bibliográfico}

O quadro a seguir exibe as principais oportunidades de pesquisa encontradas com base na análise do portfólio bibliográfico. Estas oportunidades foram descritas com base na metodologia empregadas pelos autores ou com base na definição dos próprios autores de trabalhos futuros.

Quadro 2 - Principais oportunidades de pesquisa dos artigos analisados

\begin{tabular}{|c|c|}
\hline Referência & Oportunidade de estudos futuros \\
\hline $\begin{array}{l}\text { Gaubinger e Rabl } \\
(2013)\end{array}$ & $\begin{array}{l}\text { Abordam a necessidade de se estruturar de forma detalhada as atividades do } \\
\text { Front End da Inovação, dentre elas a atividade Conceito. }\end{array}$ \\
\hline $\begin{array}{l}\text { Achiche et al. } \\
(2013)\end{array}$ & $\begin{array}{l}\text { Autores adotam a abordagem de sistema de suporte à decisão Fuzzy no FE com } \\
\text { o uso de ferramentas nas fases Identificação de Oportunidades e Análise de } \\
\text { Oportunidades. A abordagem dos autores pode ser estendida para outras fases } \\
\text { do modelo proposto por Koen, como por exemplo a fase Definição do Conceito. }\end{array}$ \\
\hline Ayağ (2016) & $\begin{array}{l}\text { Cria métodos para seleção da melhor opção de conceito dentre diversos } \\
\text { critérios, mas identifica que as imprecisões e incertezas dos gestores podem } \\
\text { influenciar na identificação dos julgamentos corretos. Propõe integrar lógica } \\
\text { fuzzy para obter resultados mais precisos. }\end{array}$ \\
\hline $\begin{array}{l}\text { Backman, } \\
\text { Börjesson e } \\
\text { Setterberg (2007) }\end{array}$ & $\begin{array}{l}\text { Os autores abordam a impossibilidade de definir um processo padrão } \\
\text { satisfatório para todos os tipos de conceitos. o contexto em que conceito está } \\
\text { inserido é fundamental para sua definição. Segundo os autores são necessárias } \\
\text { mais pesquisas sobre características da fase conceito, e assim, entender melhor } \\
\text { a sua dinâmica. }\end{array}$ \\
\hline $\begin{array}{l}\text { Cao, Zhao e } \\
\text { Nagahira (2011) }\end{array}$ & $\begin{array}{l}\text { Os autores focam sua pesquisa em teste para } 513 \text { projetos de NDP concluídos } \\
\text { em empresa de manufatura japonesas. Relatam os fatores-chave para o sucesso } \\
\text { em relação às atividades front end do NPD. Não define as atividades do FE e } \\
\text { nem o impacto de cada atividade no NDP, especialmente da atividade conceito, } \\
\text { uma vez que a fronteira para o NDP. Além disso, a pesquisa é aplicada a um } \\
\text { seguimento do mercado e apenas em empresas japonesas. }\end{array}$ \\
\hline Chew (2016) & $\begin{array}{l}\text { O artigo aborda um método chamado iSIM para fornecer inovação de serviços e } \\
\text { design de modelo de negócios para Cocriação de valor do cliente com a } \\
\text { empresa. Não foca nas atividades do FE e nem aplica o método em inovação de } \\
\text { produtos. O modelo poderia ter foco na fase de desenvolvimento de conceito } \\
\text { uma vez que aborda design de modelo de negócio. }\end{array}$ \\
\hline $\begin{array}{l}\text { Frishammar, } \\
\text { Florén e Wincent } \\
\text { (2011) }\end{array}$ & $\begin{array}{l}\text { A pesquisa foi aplicada em empresas de processos que operam na indústria de } \\
\text { metais e minerais, ou seja, fabricam produtos não montados. Segundo os } \\
\text { autores favorece o retorno de resultados potencialmente diferentes dos } \\
\text { resultados de empresas que fornecem produtos montados (prontos). Um } \\
\text { conceito focado em empresas de produtos montados poderia complementar a } \\
\text { pesquisa. }\end{array}$ \\
\hline Griffin et al. (2014) & $\begin{array}{l}\text { Esta pesquisa investiga os processos pelos quais os inovadores em série criam } \\
\text { inovações radicais através de entrevistas com } 19 \text { inovadores em série de } \\
\text { empresas consolidadas no mercado. Relatam que a pesquisa poderia ser } \\
\text { aplicada a indivíduos que trabalham em empresas iniciantes ou àqueles que } \\
\text { inovam em organizações sem fins lucrativos, como universidades ou instituições }\end{array}$ \\
\hline
\end{tabular}




\begin{tabular}{|c|c|}
\hline & $\begin{array}{l}\text { governamentais. Explorar os processos que usam para identificar problemas dos } \\
\text { clientes, inventar tecnologias e capacidades de apoio e orientar esses conceitos } \\
\text { através do processo mais formal de NPD da organização é uma possibilidade de } \\
\text { pesquisa. }\end{array}$ \\
\hline $\begin{array}{l}\text { Rocha, Souza e } \\
\text { Filho (2014) }\end{array}$ & $\begin{array}{l}\text { Outras pesquisas devem ser conduzidas em outras empresas e indústrias, para } \\
\text { verificar a adequação da estratégia de customização em massa. Adequação da } \\
\text { SBCE à customização em massa e comprovar a eficiência da prática em } \\
\text { desenvolver simultaneamente múltiplos conceitos. }\end{array}$ \\
\hline $\begin{array}{l}\text { Koen et. al. } \\
(2001)\end{array}$ & $\begin{array}{l}\text { Os autores definem a última fase do FEI como: Conceito e Desenvolvimento } \\
\text { Tecnológico, mas não detalham o conceito ou se está relacionado a um } \\
\text { processo antes do NDP. A proposta pode ser complementada acrescentando } \\
\text { um estudo mais detalhado na fase conceito. }\end{array}$ \\
\hline $\begin{array}{l}\text { Elverum e Welo } \\
(2014)\end{array}$ & $\begin{array}{l}\text { Neste artigo são estudadas as fases iniciais do NDP em sete OEMs automotivos. } \\
\text { Os autores sugerem que para se ter um conceito efetivo é necessário o } \\
\text { desenvolvimento de vários protótipos. Uma análise em outros segmentos que } \\
\text { não só no ramo automotivo poderia dar maior amplitude na pesquisa. }\end{array}$ \\
\hline $\begin{array}{l}\text { Fox } \\
(2012)\end{array}$ & $\begin{array}{l}\text { O autor descreve melhorias nos processos de triagem do NPD com uma análise } \\
\text { na fase do FEI, relacionado aos seus benefícios e as consequências negativas. } \\
\text { Esta análise poderia ser concentrada na fase conceito definindo os problemas de } \\
\text { um conceito mal formulado ou que não atenda às necessidades do } \\
\text { mercado/clientes. }\end{array}$ \\
\hline Barros et al. (2015) & $\begin{array}{l}\text { Os autores utilizam a técnica Delphi como metodologia para identificar as } \\
\text { variáveis, os principais fatores críticos que existem durante a fase de conceito do } \\
\text { produto e a influência desses fatores na definição do time-to-market. A } \\
\text { pesquisa poderia ser testada com outra técnica que não a técnica Delphi, } \\
\text { analisar e comparar os resultados. }\end{array}$ \\
\hline Bhatia et al. (2017) & $\begin{array}{l}\text { Os autores abordam a introdução de métodos ágeis para acelerar os estágios do } \\
\text { FEl com a análise principal baseada apenas na revisão de literatura. Um estudo } \\
\text { empírico com aplicação de todas as etapas, sobretudo o desenvolvimento do } \\
\text { conceito, pode dar continuidade à pesquisa. }\end{array}$ \\
\hline Duin et al. (2009) & $\begin{array}{l}\text { A proposta dos autores é a criação de um jogo para fomentar a criatividade e } \\
\text { favorecer a geração de ideias. O trabalho pode ser complementado } \\
\text { desenvolvendo métodos semelhantes para outras fases do FEl, como para } \\
\text { favorecer o desenvolvimento do conceito de um novo produto. }\end{array}$ \\
\hline
\end{tabular}

Fonte: construído pelos autores a partir do portfólio bibliográfico analisado (2018).

Como observado no quadro dois, existem lacunas que podem ser exploradas em outras pesquisas. Os diversos autores abordados no portfólio encaminharam suas pesquisas com foco no FE, como Gaubinger e Rabl (2013) que mostraram a necessidade de melhor detalhamento do FE, em especial Atividade Conceito. Para Achiche et al. (2013) o apoio à decisão dos gerentes no processo de avaliar o uso de ferramentas no FE é fator importante, para tanto, o autor aborda as atividades de Identificação de Oportunidades e Análise de Oportunidades e deixa como lacuna de pesquisa as atividades Geração de Ideias, Seleção de Ideias e Conceito passíveis de serem aplicadas o mesmo método.

Ayağ (2016) aborda que devido à imprecisão e incerteza nos julgamentos do(s) decisor (es), as escalas utilizadas ANP e TOPSIS poderiam ser insuficientes e imprecisas para captar os julgamentos corretos do (s) gestor (es). Portanto, para pesquisas futuras o autor recomenda que uma lógica fuzzy pode ser integrada a essa abordagem para obter resultados mais satisfatórios na atividade conceito.

No artigo de Backman, Börjesson e Setterberg (2007) fica claro para próximos trabalhos que existe a necessidade de mais pesquisas sobre características do conceito, e assim, entender melhor a dinâmica nesta atividade. Já em Cao, Zhao e Nagahira (2011) é sugerido para os estudos do FE devem ser estendidos a diferentes setores da indústria.

Chew (2016) desenvolve um novo método de projeto integrado conhecido como iSIM (Integrated Service Innovation Method) para projetar um novo modelo de serviço e negócios. A lacuna a ser explorada 
neste artigo está na criação de um modelo ontológico formal do método para o desenvolvimento de uma ferramenta de software iSIM.

Em Elverum e Welo (2014) os resultados deste artigo são limitados a sete empresas da indústria automotiva, e pesquisas futuras devem se expandir para outros tipos de indústrias e talvez considerar estudos envolvendo uma amostra mais ampla e métodos quantitativos para obter dados mais concretos.

No trabalho de Frishammar, Florén e Wincent (2011) é possível que outros estudos em inovação de processos sejam feitos e testados em uma amostra mais ampla de empresas e em outros domínios industriais, preferencialmente com uma pesquisa em larga escala empregando técnicas multivariadas para testar hipóteses formais sobre o papel da incerteza e da equivocidade em projetos front end da inovação.

Observa-se no trabalho de Barros et al. (2015) que o estudo de caso coletou dados de projetos já entregue no NPD, mas é importante ser explorado a realização do modelo proposto no início de novos projetos para identificar os possível influência dos fatores críticos nas atividades anteriores ao conceito ou a criação de um novo produto.

Todos os Inovadores entrevistados na pesquisa de Griffin et al. (2014) trabalharam em empresas maduras com fins lucrativos, os resultados deste estudo podem não ser aplicáveis a indivíduos que trabalham em empresas iniciantes ou àqueles que inovam em organizações sem fins lucrativos, como universidades ou instituições governamentais. Uma possibilidade de pesquisa é aplicar o método em empresas pequenas ou sem fins lucrativos.

A lacuna de pesquisa no trabalho de Rocha, Souza e Filho (2014) tem relação aos dados e análises que foram realizados em uma única empresa. Portanto, outras pesquisas devem ser realizadas em outras empresas e indústrias seguindo a mesma metodologia.

No material de Koen et. al. (2001) tem a definição da atividade conceito, mas não está claro se existem etapas relacionadas à esta atividade. Trabalhos futuros podem explorar esta atividade.

Bhatia et al. (2017) voltaram seus estudos para a análise na revisão de literatura. A pesquisa poderia ser complementada um estudo empírico, pois envolverá experiências reais e coleta de dados primários que podem ser usados para analisar e compreender impacto desta integração na eficácia da gestão do FEI.

\section{CONCLUSÃO}

Esta é uma pesquisa preliminar que apontou o resultado de uma amostra de trabalhos seguindo a metodologia empregada e não destinada a esgotar o assunto. Entretanto, esta amostra foi significativa para se traçar um panorama geral acerca do que se vem discutido sobre a fase conceito no front end da inovação.

Como observado neste trabalho, as publicações relacionadas ao assunto vêm aumentando conforme os anos, tendo seu volume maior no ano de 2016 com quatro publicações. Neste sentido, identificou-se que a predominância dos trabalhos de natureza qualitativa representando um total de $70 \%$ dos casos classificados, ao passo que $20 \%$ são de natureza quantitativa e $10 \%$ utilizaram a abordagem mista. Na outra análise foi apresentado os países que publicaram e auxiliam a entender como está distribuída a pesquisa sobre o assunto ao redor do mundo, contudo, observou-se que o Estados Unidos foi o país que mais publicou, com total de 5; Brasil, Alemanha, Noruega e Suécia, com total de duas; Turquia, Israel, Japão, Irlanda, Finlândia, Dinamarca e Austrália, com uma publicação cada. Desta maneira, verifica-se que as pesquisas sobre este assunto ainda estão concentradas em poucos países.

Outro fator analisado neste trabalho foi o estrato do site ELSEVIER (2019). Para essa análise, cada um dos 20 periódicos, bem como os respectivos fatores de impacto e índice $\mathrm{H}$, foram averiguados visando identificar a qualificação das revistas. Percebeu-se que metade das publicações (10) resultaram de envios a conferências, sendo assim não possuem qualificação pelo SJR. Os artigos com qualificação Q1 e Q2 são os que publicaram em periódicos da área de Negócios e Gestão, Indústria e Engenharia de Manufatura, Estratégia e Gestão, Marketing e Sistemas de Informação, totalizando sete publicações. Duas publicações fazem parte de um livro específico sobre a estruturação do Front End e dessas, apesar de não ter qualificação no SJR, obtevese uma grande contribuição para atender os objetivos da pesquisa. Somente uma publicação teve qualificação Q4, mas isso se deve ao fato de ser um periódico novo. 
Os autores abordados neste material tratam a atividade conceito de diversas formas, mas fica claro em todos os trabalhos que esta atividade tem sua importância, especialmente no impacto causado na próxima fase do processo de inovação. Desta maneira foi possível observar possibilidades de pesquisas nos trabalhos analisados, seja para dar continuidade, seja para abordar aspectos que não faziam parte do escopo deste trabalho. Aumentar o número de trabalhos que abordam o tema de maneira quantitativa é uma oportunidade de pesquisa que pode enriquecer as discussões sobre o assunto e explorar lacunas que ainda não estão bem definidas.

Outra conclusão deste trabalho está relacionada ao fato que muitas pesquisas analisadas definem fases para o Front End Inovação, mas não identifica fases para atividade conceito. Entender esta atividade e analisar subdivisões que ela possa apresentar, ou o fluxo de seu processo que define a qualidade para desenvolvimento de novos produtos, pode tornar o processo de inovação mais assertivo.

\section{REFERÊNCIAS}

ACHICHE, S. et al. Fuzzy decision support for tools selection in the core front end activities of new product development. Research in Engineering Design, v. 24, n. 1, p. 1-18, 2013.

ANTONELLO, M. G.; ROMANO, L. N.; MARTINS, M. E. S. A importância do processo de sistematização de conhecimentos para o desenvolvimento de produtos. Revista Espácios, v. 36, n. 5, p. 1-12, 2015.

AYAĞ, Z. An integrated approach to concept evaluation in a new product development. Journal of Intelligent Manufacturing, v. 27. n. 5, p. 991-1005, 2016.

ARTTO, K. et al. The integrative role of the project management office in the front end of innovation. International Journal of Project Management, v. 29, n. 4, 2011, p. 408-421

BACKMAN, M.; BÖRJESSON, S.; SETTERBERG, S. Working with concepts in the fuzzy front end: Exploring the context for innovation for different types of concepts at Volvo Cars. R \& D Management, v. 37, n. 1, p. 17-28, 2007.

BAREGHEH, A.; ROWLEY, J.; SAMBROOK, S. Towards a multidisciplinary definition of innovation. Management Decision, v. 47, n. 8, p. 1323-1339, 2009.

BJÖRK, J., BOCCARDELLI, P.; MAGNUSSON, M. G. Ideation capabilities for continuous innovation. Creativity and Innovation Management, v. 19, n. 4, p. 385-396, 2010.

BHATIA, A. et. al. Improving the effectiveness of fuzzy front end management: Expanding stage-gate methodologies through agile. In: PORTLAND INTERNATIONAL CONFERENCE ON MANAGEMENT OF ENGINEERING AND TECHNOLOGY (PICMET), Portland, EUA. Proceedings [...]. Portland: PICMET, 2017. p. 1-8.

BARROS, M. V. et al. Analysis of time to market complexity: A case study of application of Bayesian networks as a forecasting tool. In: INTERNATIONAL CONFERENCE ON INDUSTRIAL ENGINEERING AND SYSTEMS MANAGEMENT (IESM), Seville, Spain 2015. Proceedings [...]. Seville: IESM, 2015. p. 1197-1204.

CAO, Y.; ZHAO, L.; NAGAHIRA, A. The impact of front end innovation in new product development in Japanese manufacturing companies. Nankai Business Review International, v. 2, n. 1, p. 98-113, 2011.

CAREY, K.; HELFERT, M. Improving the front end of innovation: The case of mobile commerce services. In: INTERNATIONAL CONFERENCE ON HCI IN BUSINESS, GOVERNMENT, AND ORGANIZATIONS: ECOMMERCE AND INNOVATION, 3., Toronto, Canada. Proceedings [...]. Toronto: HCIBGO, 2016. p. 491-501.

CHEW, E. K. iSIM: An integrated design method for commercializing service innovation. Information Systems Frontiers, v. 18, n. 3, p. 457-478, 2016.

DUIN, H. et al. A concept for serious gaming to support disruptive idea generation. In: IEEE INTERNATIONAL TECHNOLOGY MANAGEMENT CONFERENCE (ICE), Leiden, 2009. Proceedings [...]. Leiden: IEEE. 2009. p. 1-9. 
ELFVENGREN, K.; KORTELAINEN, S.; TUOMINEN, M. Managing the front end of innovation with a group support system. International Journal of Entrepreneurship and Innovation Management, v. 10, n. 3-4, p. 266-279, 2009.

ELSEVIER. SRJ Scimago Journal \& Country Rank. Disponível em: https://www.scimagojr.com/. Acesso em: 29 ago. 2019.

ELVERUM, C. W.; WELO, T. The role of early prototypes in concept development: Insights from the automotive industry. In: CIRP DESIGN CONFERENCE, 24., 2014, Milano. Proceedings [...]. Milano, Italy: Elsevier, 2014. p. 491-496.

FLINT, D. J. Compressing new product success-to-success cycle time: deep customer value understanding and idea generation. Industrial Marketing Management, v. 31, n. 4, p. 305-315, 2002.

Fox, Stephen. Improving new product development by screening manufactured goods for potential disbenefits and negative consequences. Journal of Manufacturing Technology Management, v. 23, n. 5, p. 615-633, 2012

FRISHAMMAR, J.; FLORÉN, H.; WINCENT, J. Beyond managing uncertainty: Insights from studying equivocality in the fuzzy front end of product and process innovation projects, IEEE Transactions on Engineering Management, v. 58, n. 3, p. 551-563, 2011.

FRISHAMMAR, J. et al. The Front End of Radical Innovation: A Case Study of Idea and Concept Development at Prime Group. Creativity and Innovation Management, v. 25, n. 2, p. 179-198, 2016.

GAUBINGER, K.; RABL, M. Structuring the front end of innovation. In: GASSMANN, O.; SCHWEITZER, F. (org.) Management of the fuzzy front end of innovation. Suiça: Springer, 2013. p. 15-30.

GASSMANN, O.; SCHWEITZER, F. Fuzzy front end of innovation: Quo Vadis? In: GASSMANN, O.; SCHWEITZER, F. (org.). Management of the Fuzzy Front End of Innovation. Suiça: Springer, 2013. p. 301-310.

GRIFFIN, A. et al. Serial Innovators processes: How they overcome barriers to creating radical innovations. Industrial Marketing Management, v. 43, n. 8, p. 1362-1371, 2014.

HERSTATT, C.; VERWORN, B. The" fuzzy front end" of innovation. Working Papers/Technologie-und Innovations management. Hamburg: Technische Universität Hamburg-Harburg, 2001.

HERSTATT, C. et al. A. Fuzzy front-end practices in innovating japanese companies. International Journal of Innovation and Technology Management, v. 3, n. 1, p. 43-60, 2006.

ILUZ, M.; SHTUB, A. Simulation based planning of the fuzzy front-end stage of a project. In: DESIGN CONFERENCE; INNOVATIVE PRODUCT CREATION, 36., 205, Haifa. Proceedings [...]. Haifa, Israel: Elsevier. p. 106-110.

JEVNAKER, B. H.; TELLEFSEN, B.; LÜDERS, M. Front-end service innovation: Learning from a design-assisted experimentation. European Journal of Innovation Management, v. 18, n. 1, p. 19-43, 2015.

KIM, Jongbae; WILEMON, David. Strategic issues in managing innovation's fuzzy front-end. European Journal of Innovation Management, v. 5, n. 1, p. 27-39, 2002.

KOEN, P. A. et al. Providing clarity and a common language to the "fuzzy front end". Research Technology Management, v. 44, n. 2, p. 46-55, 2001.

KOEN, P. A.; BERTELS, H.M. J; KLEINSCHMIDT, E. J. Managing the Front End of Innovation-Part II: Results from a Three-Year Study: Effective Front-End activities were found to be significantly different for incremental and radical projects. Research-Technology Management, v. 57, n. 3, p. 25-35, 2014.

ROCHA, H. M.; SOUZA, C. N. A.; FILHO, D. F. S. Mass Customization Enablement Through Lean Design \& SetBased Concurrent Engineering Application. JOSCM: Journal of Operations and Supply Chain Management, v. 7, n. 2, p. 124-139, 2014. 
SALMELA, E.; SANTOS, C.; HAPPONEN, A. Formalization of front-end innovation in supply network collaboration. International Journal of Innovation and Regional Development, v. 5, n. 1, p. 91-111, 2013.

SANDMEIER, P. et al. Towards a structured and integrative Front-end of product innovation. In: R\&D MANAGEMENT CONFERENCE (RADMA), 2004, Lisboa. Proceedings [...]. Lisboa: ISPA, 2004. p. 1-9.

STEVENS, E. Fuzzy front-end learning strategies: Exploration of a high-tech company. Technovation, v. 34, n. 8, p. 431-440, 2014.

STEVENS INSTITUTE OF TECHNOLOGY. A Benchmarking Study of Front End of Innovation Activities and Performances. 2004. Disponível em: http://www.stevens-tech.edu/cce/ NEW/PDFs/ Survey_Quetsions_ 051304.pdf. Acesso em: 11 fev. 2019.

BREM, A.; VOIGT, K. Integrated Idea Management in Emerging Technology Ventures. International Journal of Technology Policy and Management, v. 7, n. 3, p. 211-215, 2007.

TAKAHASHI, S.; TAKAHASHI, V.P. Gestão de inovação de produtos: estratégia, processo, organização e conhecimento. São Paulo: Elsevier, 2007.

TASCA, J. E. A contribuição da avaliação de desempenho, como um instrumento de apoio à decisão, para a prevenção ao crime baseada no ambiente. Tese (doutorado) - Universidade Federal de Santa Catarina, Centro Tecnológico, Programa de Pós-Graduação em Engenharia de Produção, Florianópolis, 2013. $350 \mathrm{p}$.

THANASOPON, B.; PAPADOPOULOS, T.; VIDGEN, R. Opening Up The Fuzzy Front-End Of Service Process Innovation: Searching Capability, Co-Development Capacity, And IT Competence. In: EUROPEAN CONFERENCE ON INFORMATION SYSTEMS (ECIS), Utrecht, Netherlands, 2013. Proceedings [...]. Utrecht: Utrecht University, 2014, p. 1-7.

TIDD, J.; BESSANT, J. Gestão da Inovação. 5. ed. Porto Alegre: Bookman, 2015.

ZHAO, L.; XIANG, Y.; YI, Q. Fuzzy front-end patent management and innovation performance: mediating role of patent commercialization and moderating effect of technological lock-in. Management Decision, v. 55, n. 6, 1143-1162, 2017. 